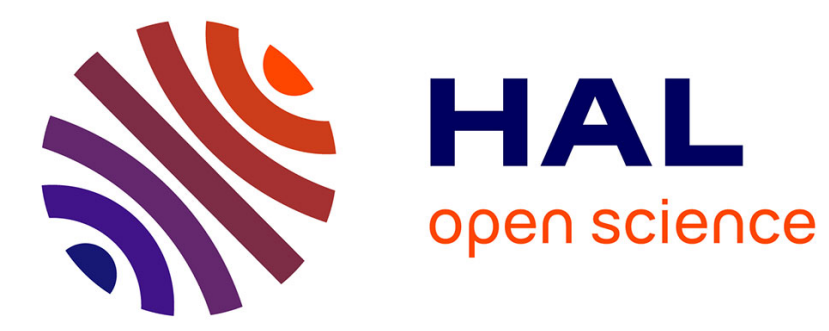

\title{
Users' Behavioral Strategies Toward Mobile App Problems: Fight or Flight
}

\author{
Yavuz Inal, Tuna Hacaloglu
}

\section{To cite this version:}

Yavuz Inal, Tuna Hacaloglu. Users' Behavioral Strategies Toward Mobile App Problems: Fight or Flight. 18th Conference on e-Business, e-Services and e-Society (I3E), Sep 2019, Trondheim, Norway. pp.37-49, 10.1007/978-3-030-29374-1_4. hal-02510124

\section{HAL Id: hal-02510124 \\ https://hal.inria.fr/hal-02510124}

Submitted on 17 Mar 2020

HAL is a multi-disciplinary open access archive for the deposit and dissemination of scientific research documents, whether they are published or not. The documents may come from teaching and research institutions in France or abroad, or from public or private research centers.
L'archive ouverte pluridisciplinaire HAL, est destinée au dépôt et à la diffusion de documents scientifiques de niveau recherche, publiés ou non, émanant des établissements d'enseignement et de recherche français ou étrangers, des laboratoires publics ou privés. 


\title{
Users' Behavioral Strategies toward Mobile App Problems: Fight or Flight
}

\author{
Yavuz Inal ${ }^{1}$ and Tuna Hacaloglu ${ }^{2}$ \\ ${ }^{1}$ Department of Information Science and Media Studies, \\ University of Bergen, Bergen, Norway \\ yvzinal@gmail.com \\ ${ }^{2}$ Information Systems Engineering, \\ Atilim University, Ankara, Turkey
}

\begin{abstract}
In this paper, we identify two distinct behavioral strategies for dealing with problems encountered in the use of mobile apps - fight or flight. In the fight strategy, individuals do not give up using an app when faced with a problem; rather, they experiment with different ways to cope with that problem, whereas the flight strategy refers to the user's decision to uninstall an app when they encounter a problem and/or their intention to use an alternative app. These strategies were identified from an analysis of documents, which forty-two users reported, and can be used to understand how users deal with encountered problems. The participants were asked to use a mobile app of their choice for one week and report the behavioral strategies they utilized to counter problems they experienced. According to the findings obtained from content analysis, the most reported complaints concerned the categories of interface design, functional error, feature request, and feature removal. The participants who complained about functional errors, frustrating features, and slow application speed stopped using the app (flight behavior) whereas those that were dissatisfied with the interface, a missing feature or the content of the app continued to use the app and tried to overcome the problems (fight behavior).
\end{abstract}

Keywords: User Behavior, Behavioral Strategy, Human-Computer Interaction, Mobile Interface Design, Mobile Apps, Fight or Flight.

\section{Introduction}

With rapid developments in technology, many new concepts have emerged in recent years to replace the term "computer" in the context of human-computer interaction. Mobile devices, being one of the technologies that people are most interacting with today, have an important place in this interaction [1]. Beyond communication, gaming or entertainment, it is possible to see mobile technologies in many areas from electronic commerce, banking and public services to information and communication systems [2]. Today, people have even begun to fear the absence of mobile phones, and there is a 
positive relationship between this fear and the duration of mobile device use [3]. Therefore, the analysis and evaluation of user behavior toward mobile apps, which are active parts of everyday life, play an essential role in the success of apps [4].

Due to the growing popularity and trends toward widespread adoption of mobile devices, in recent years millions of apps have been developed for these devices. For example, according to Statista data, as of March 2017, there are 2.8 million apps for Android devices in Google Play Store and 2.2 million apps for iOS devices in Apple Store [5], and there is a greater adoption of smartphones and other mobile devices than PCs [6]. Thus, mobile apps have become the fastest growing part of the software world [7], and mobile app development continues to increase in popularity as an important area of work for software developers. Today, with the "Mobile-First" trend, software developers are expected to develop apps first for the mobile platform and then for computers [8]. However, mobile devices also have significant limitations, particularly concerning screen size, limited processing capacity, different design requirements, and the context in which they are used $[9,10]$. Given all these limitations, it is clear that software developers need to pay more attention to the needs and expectations of users when developing mobile apps than desktop apps. In addition, the features of desktop apps and those of mobile apps significantly differ. These limitations and differences have also increased the importance of evaluating mobile apps [11].

Feedback and the evaluation by mobile app users are important sources of information $[12,13]$. Studies have shown that features of mobile apps are a determinant of user behavior toward these apps [14-16]. User complaints regarding both the functional characteristics of an app [14] and its design and aesthetic appearance have a considerable influence on the user's decision to continue using it [17].

This study aimed to determine the problems encountered in mobile apps and the behavioral strategies adopted by users to overcome these problems. The data were collected from university students, who were active mobile app users. The findings of the study are expected to offer guidance to not only researchers but also mobile app developers and consultants.

Primary research questions of the study were addressed as below,

- What are the usability problems that the participants encountered while using mobile apps?

- What are the participants' behavioral strategies against the encountered mobile app problems?

\section{Related Works}

Mobile devices are one of the most important technological innovations of today [18]. With rapid evolution of these devices, mobile apps are gaining increasingly more attention [19]. Therefore, an important area of research is the identification of problems faced by users when using mobile apps and the analysis of the behavioral strategies they develop to counter these problems. However, in the literature, there are very few studies related to the assessment of user behavior, attitudes or their evaluation concerning mobile apps [20]. 
Chou et al. [21] examined the behavior and habits of users toward mobile apps in the context of expectance-confirmation theory. The authors gathered questionnaire data from university students and determined that the ultimate success of an app is related to the continued use of that app. In addition, they concluded that mobile apps that were considered useful and enjoyable positively influenced user satisfaction and users' tendency to continue using those apps. Similarly, Hsu and Lin [4] analyzed users' purchasing behavior of paid mobile apps based on the expectation confirmation model. The data collected from 507 users through a questionnaire showed that confirmation was positively associated with perceived value and satisfaction of users; therefore, the authors emphasized that confirmation was an important factor in using mobile apps. In addition, they determined that positive feedback given by other users for a mobile app had a positive effect on the purchasing behavior related to that app.

In another study [16] that aimed to determine mobile users' purchasing and information sharing behaviors, the effect of differences of the mobile platform, user interest in mobile apps, and last visits to mobile stores were investigated. The data was collected from 345 participants through a questionnaire. At the end of the study, it was reported that interest in a mobile e-commerce app was positively related to users' purchasing and information sharing behaviors. Wang et al. [22] focused on the factors that affect users' mobile app use based on the role of consumption values. The data was obtained from 282 mobile app users. It was found that functional, social, emotional and epistemic values had an important effect on the development of behaviors concerning app use. Similarly, Chang et al. [15] evaluated 12 different mobile apps in their analysis of factors that affect decisions to use mobile apps. The data gathered from 68 participants through a questionnaire revealed that users' needs and excitement regarding an app as well as its usability were influential factors in their decision to download and use that app.

Maghnati and Ling [23] conducted a study to determine the effect of experiential value on user attitudes toward mobile apps. User attitudes were examined under the experiential value categories of aesthetic, playfulness, service excellence, and customer return on investment. User attitudes were found to have a significant positive relationship with playfulness and customer return on investment, but not with aesthetic and service excellence. Similarly, Le and Nguyen [24] explored the effect of advertisements in mobile apps on user attitudes based on data collected from 206 participants using a questionnaire. Although many users were negative about advertisements in mobile apps, it was considered that the format and content of advertisements could be designed in a way that would appeal to users. In this context, the authors determined that "credibility" and "entertainment" in advertisements were the main factors that affected user attitudes toward advertisements.

Features of mobile apps are determinants of not only the attitudes of users toward these apps, but also the development of user perceptions and preferences. For example, Kim et al. [25] focused on the effects of "stickiness" and "word of mouth" on user perceptions concerning mobile app features. As a result of a survey conducted with 503 smartphone users, it was determined that user perceptions toward mobile app features were positively associated with the usability of apps. Furthermore, this resulted in increased "stickiness" and positive "word of mouth" intentions. Huy and vanThanh [26] 
identified the most popular mobile app paradigms and evaluated them from the perspective of developers, users, and service providers. From the users' point of view, it was determined that ease-of-use and functionality were very important in native mobile apps. In another study focusing on users' perspective on mobile apps, Bowen and Pistilli [14] investigated university students' preferences concerning the use of mobile apps. A total of 1,566 students studying at Purdue University were reached through a questionnaire. According to the data obtained, a significant number of participants used an Android phone or iPhone. The participants considered themselves to be moderate and advanced users, and were found to prefer native mobile apps because they are quicker and easier to use than the mobile Web.

User evaluation of mobile apps is critical to obtaining valuable information about the current state of apps, their place in the market, and their success. In this context, in addition to the feedback received from users through face-to-face interviews or questionnaires, user evaluation of apps in app stores provides important information [27]. Therefore, while some studies that analyzed user data in these environments used a manual analysis method, other studies proposed an automated evaluation system. For instance, Khalid et al. [27] investigated the most frequent complaints of users in relation to mobile apps. The authors manually reviewed a total of 6,390 user ratings for the 20 most popular iOS apps. The problems most users complained about were functional errors, feature requests, and app crashes. Fu et al. [13] proposed a system called WisCom to analyze user reviews of mobile apps. The authors stated that this system provided valuable information about the entire mobile app market by identifying inconsistencies in user ratings, the reasons why users like or dislike an app, and user preferences of different mobile apps.

\section{$3 \quad$ Methodology}

A total of 42 undergraduate university students enrolled in the Software Engineering, 29 male and 13 female, participated in the study. Different methods and techniques have been used in the research involving user evaluation of mobile apps. Some studies analyze feedback from users [e.g., 16, 25] while others assess user ratings on apps in app stores [e.g., 27]. In this study, descriptive quantitative analysis was used to engage in an in-depth analysis of user evaluation of mobile apps and to identify the adopted behavioral strategies.

\subsection{Data Collection Procedure}

It takes approximately eight minutes for users to learn how to use a new mobile app [28]. A large percentage of users decide whether to remove an app from their smart devices within three to seven days of first using that app [29]. In this context, the oneweek timeframe from the moment that users learn how to use a mobile app is very critical for the success of that app in the market. This one-week period of use is considered to be sufficient to obtain the information necessary to evaluate a mobile app. Therefore, the participants in this study were given an assignment in a Human Computer Interaction Course, and asked to use a mobile app of their choice for one week. 
Then, they reported in detail the problems they encountered during the use of the app, as well as the behavioral strategies they adopted to counter these problems. The participants reported name of the mobile application that they evaluated, list of the usability problems that they countered, and their decisions regarding these problems in a document. The participants documented these issues as they happened and the reports were written using a word processor, and the participants sent their documents to the researchers via e-mail. The basic data used in the study was obtained through the descriptive quantitative analysis of the participants' documents.

Reaction to a problem in an app might depend on the type of app and users ' needs. Users may have different tolerance towards various apps. Therefore, although the participants were completely free to choose the mobile app to evaluate, only free and hedonic-oriented apps were evaluated in the study because this may influence the features that an app offers as well as the participants' decision to uninstall it. Hedonic-oriented apps are type of mobile applications that are used for enjoyment, arousal and freedom [30]. This allowed for the coverage of a broader range of mobile apps through the assessment of different themes and types of hedonic-oriented apps and ensuring that the implications of research were more generalizable under this type of apps. Therefore, the types of mobile apps evaluated by the participants varied under 7 different themes such as photography, video, chat, sports, music, news and games.

\subsection{Data Analysis}

A content analysis was performed on the qualitative data collected from the participants. The mobile app problems reported by the participants were categorized using the 12 types of complaints determined by [27] based on user feedback (see Table 1). Each mobile app problem identified by the participants was included in one of these 12 categories and analyzed accordingly.

Table 1. Type of complaints identified by [27] and their description (p.74)

\begin{tabular}{ll}
\hline Type of Complaint & Description \\
\hline App Crashing & The app often crashed. \\
Compatibility & The app had problems on a specific device or an OS version. \\
Feature Removal & A disliked feature degraded the user experience. \\
Feature Request & The app needed additional features. \\
Functional Error & The problem was app specific. \\
Hidden Cost & The full user experience entailed hidden costs. \\
Interface Design & The user complained about the design, controls, or visuals. \\
Network Problem & The app had trouble with the network or responded slowly. \\
Privacy and Ethics & The app invaded privacy or was unethical. \\
Resource Heavy & The app consumed too much energy or memory. \\
Uninteresting Content & The specific content was unappealing. \\
Unresponsive App & The app responded slowly to input or was laggy overall. \\
\hline
\end{tabular}


Furthermore, behavioral strategies adopted by participants to handle the problems they identified were grouped into either 'fight' (continued to use) or 'flight' (abandoned the app). This made it possible to demonstrate the relationship between the problems encountered in mobile apps and the behavior exhibited. In the 'fight' strategy, individuals do not give up using an app when faced with a problem; rather, they experiment with different ways to cope with that problem. On the contrary, the flight strategy refers to the user's decision to uninstall an app when they encounter a problem and/or their intention to use an alternative app. Each mobile app problem included in one of the 12 complaint types were categorized as fight or flight.

\section{$4 \quad$ Results}

\subsection{Problems Identified in Mobile Apps}

Figure 1 presents the problems identified by the participants for the mobile apps they evaluated. Most of the participants complained about the interface design of mobile apps (Interface Design, $\mathrm{n}=32,76 \%$ ). This category contained complaints, such as the app not being attractive or aesthetic, use of wrong color contrast, problems with size and alignment of images, font selection, and readability of texts. Visual design issues that affected the ease-of-use of the app were also included in this category. Functional errors in the app constituted another type of problems according to most of the participants (Functional Error, $n=29,69 \%$ ). Incorrect operation of buttons, certain operations giving constant errors, and some pages not responding were examples of this type of complaint.

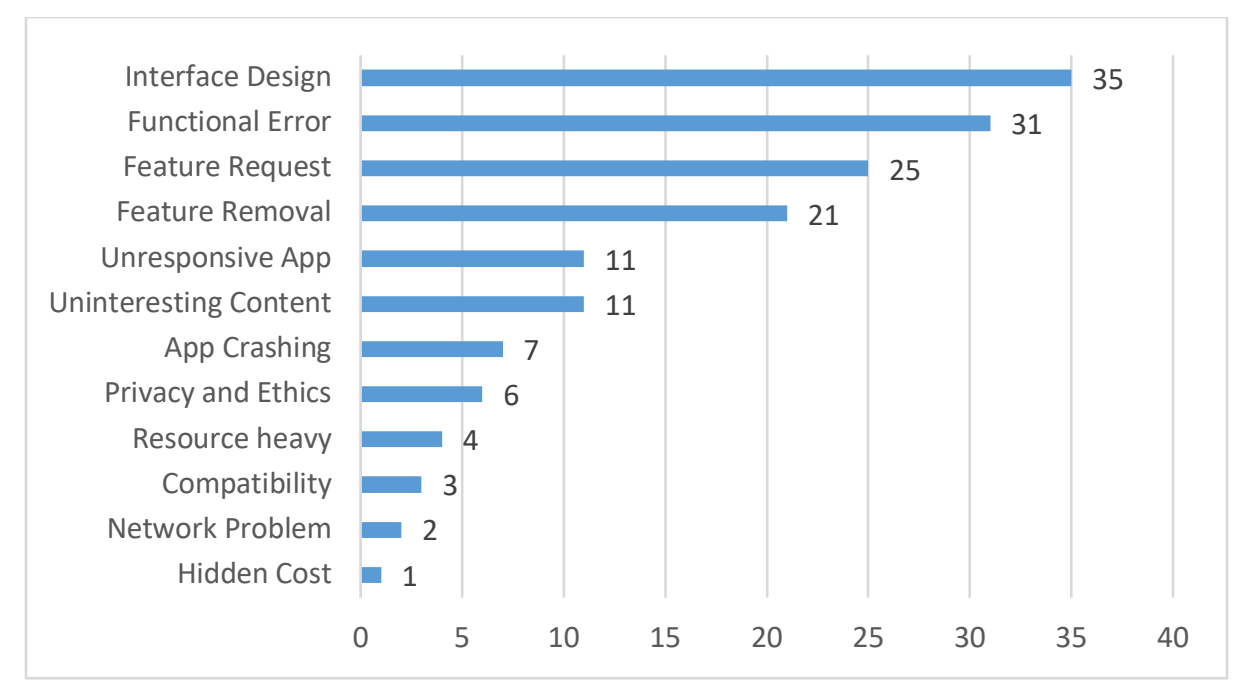

Fig. 1. Mobile app problems reported by the participants (percentage) 
As the number of apps developed for mobile devices increases, user expectations and demands from these apps also increase. In this study, it was found that more than half of the participants complained about features they thought were missing in the mobile apps they evaluated (Feature Request, $\mathrm{n}=21,50 \%$ ). The lack of an option to close a bank account, problems with bank integration, short duration of video recording, or lack of feedback within the app were among the reported issues.

Some of the participants were frustrated by the services, content or operations offered by the mobile apps (Feature Removal, $n=18,43 \%$ ), and they complained about the requirement to update the app, constant advertisements displayed in the app that forced the user to make a payment to close the window, and the recommendation to contact the management rather than describing the error in error messages.

The participants also referred to problems regarding the slow application speed generally, or in certain pages or operations, particularly on certain days of the week (Unresponsive App, $n=10,24 \%$ ). It is important that the content in apps is consistent with the features of the app and user expectations. However, concerning some of the mobile apps evaluated in this study, users had complaints regarding this situation (Uninteresting Content, $\mathrm{n}=9,21 \%$ ). Examples given by the participants under this type of complaint were the app not presenting information that was needed and the content being limited and inadequate or including irrelevant information. Other mobile app issues identified by the participants include app crashes (App Crashing, $n=4,10 \%$ ), excessive use of battery, memory or internet (Resource Heavy, $n=4,10 \%$ ), violation of privacy and ethics (Privacy and Ethics, $n=3,7 \%$ ), and problems concerning the hardware or software specifications required by the app (Compatibility, $\mathrm{n}=2,5 \%$ ).

\subsection{Behavioral Strategies Adopted to Counter the Problems}

The behavioral strategies developed by participants to counter the problems they faced when using the mobile apps were analyzed in detail. It was determined that the participants either responded by trying to find solutions to these problems (Fight) or abandoning the use of app and searching for an alternative (Flight). The methods used by the participants to cope with the problems are detailed in Table 2 according to the behavioral strategy.

Table 2. Behavioral strategies adopted by users to handle mobile app problems

\begin{tabular}{llll}
\hline & Users' Behavioral Strategies & $\mathbf{n}$ & \% \\
\hline \multirow{4}{*}{ Fight } & Making an additional effort using the trial and error method & 23 & 29 \\
& Updating or reinstalling the app & 9 & 12 \\
& Sending feedback to the app developer(s) & 5 & 6 \\
& Contacting the customer services & 5 & 6 \\
& Seeking help within the app & 2 & 3 \\
\hline \multirow{3}{*}{ Flight } & Uninstalling the app & 12 & 15 \\
& Using the app less & 9 & 12 \\
& Seeking an alternative app & 7 & 9 \\
& Using the website of the app & 6 & 8 \\
\hline
\end{tabular}


The participants seemed to mostly prefer to make an additional effort to perform the operations they needed in the mobile app or to try to determine the causes of problems that confused them so that they would not trigger the same error in future use $(50 \%, \mathrm{n}$ $=21)$. This was followed by the behavior of updating or reinstalling the app $(17 \%, \mathrm{n}=$ 7). Other methods adopted by participants to tackle the app-related problems included sending feedback or complaining to the developer $(12 \%, \mathrm{n}=5)$, contacting the customer services if it was an official app of a corporation $(7 \%, n=3)$, and searching for a help feature within the app $(2 \%, \mathrm{n}=1)$.

Among the participants that adopted the behavior to abandon the app after facing problems, most preferred to uninstall the app $(26 \%, n=11)$. This was followed by reduced use of the app $(19 \%, \mathrm{n}=8)$. The participants also reported that if they thought that the mobile apps, they were trying did not meet their expectations, they would use an alternative app designed for the same purpose to fulfill their needs $(14 \%, n=6)$. Finally, some of the participants stated that they resorted to the website version to perform the actions that were problematic on the mobile app $(10 \%, n=4)$.

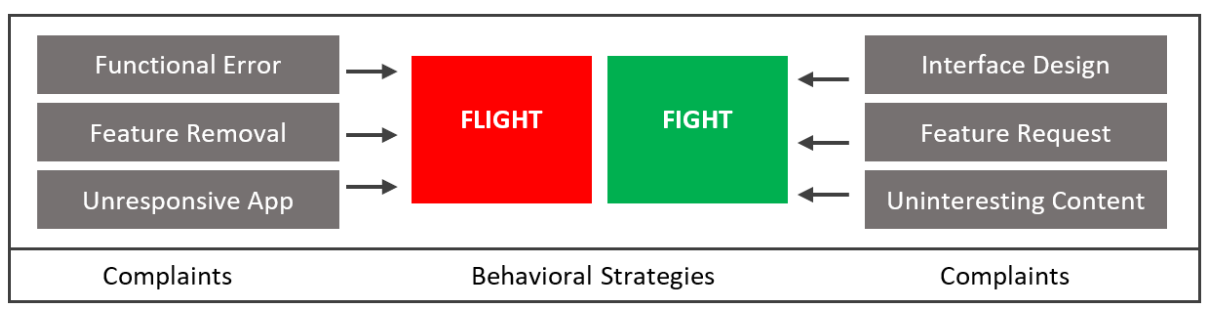

Fig. 2. Relationship between mobile app problems and behavioral strategies

In this study, the relationship between the mobile app problems identified and the behavioral strategies developed by the participants for these problems was also analyzed (see Fig. 2). The most reported complaints were considered to understand the participants ' behavioral strategies properly, and rests such as app crashing, privacy and ethics, compatibility were ignored. It was found that the variety of the problems encountered during the use of mobile apps resulted in the differentiation of methods and behavioral strategies adopted to cope with these problems. For example, the majority of the participants that complained about functional errors (23 of $29,79 \%$ ), feature removal (11 of 18,61\%), or an unresponsive app (6 of 10,60\%) adopted the flight behavior by deciding not to use the app any more. On the other hand, those participants that were not happy with the interface design, ease-of-use, aesthetics (Interface Design, 25 of $32,78 \%$ ), lack of features (Feature Request, 15 of $21,71 \%$ ), or app content (Uninteresting Content, 5 of 9, 56\%) exhibited the fight behavior, continuing to use the app and trying to overcome the problems. Other types of complaints addressed by the participants were not concentrated under any distinct behavioral strategy. 


\section{Discussion}

The development of user-friendly mobile apps is a challenging process [31]. In addition to their unique features, mobile apps have many limitations [32, 33]. However, given the growing interest in mobile apps, competition in the industry, and the expectations of users, it is important that apps have features that will minimize user complaints.

\subsection{Complaints about the evaluated mobile apps}

According to the results of the present study, the participants most complained about the interface design of mobile apps. They frequently emphasized problems concerning the aesthetic design and ease-of-use of the apps they evaluated. Mobile apps should be easy to use by target groups [34]. Individuals that do not have any difficulty using an app usually consider it user-friendly [35]. Research has shown that users assess the interfaces of mobile apps mostly based on their visual appearance [17], and the easeof-use of an app is a decisive factor for preference of use $[14,26]$. In this context, difficulty to understand how to use an app for the first time, uninteresting design, and visual problems are among issues to be considered in the development of mobile apps.

When mobile apps are being developed, they should be tested concerning different aspects, such as hardware, screen size, platform, and network connectivity [36, 37]. This will prevent potential users from encountering functional errors. However, considering that many developers manually test mobile apps due to the lack of automated testing tools for mobile apps and user testing [38], it is inevitable that users will experience functional problems in mobile apps. Huy and vanThanh [26] underlined the importance of the functionality of mobile apps from the point of view of users. Khalid et al. [27] determined that the most common complaints of users concerning mobile apps were functional errors. Similarly, in the current study, most of the participants complained about the functional problems they encountered in mobile apps.

In this study, some of the participants were also frustrated by and complained about some features or content of the mobile apps they evaluated, particularly in relation to the advertisements placed in apps. Advertisements are an important source of income for mobile app developers [39]. However, attention should be paid not to place advertisements in areas that would make it difficult for users to interact with the app and would interfere with their use of the app. Displaying a large number of advertisements is one of the main factors that negatively affects users' attitude toward an app [40]. Complex interaction provided by apps was another feature that displeased the participants. Mobile apps offer more interactivity with users compared to desktop apps [41], but it is important that this interaction is structured in a way that users can easily understand. Otherwise, users will not be able to use mobile app effectively.

In their research involving user evaluation of the usability of mobile apps, Fung et al. [42] found that the most frequent problems were inconsistencies regarding the content, the illogical presentation of information, and the lack of sufficient help concerning error messages. Similarly, among other problems, the participants reported that some of the necessary information was not included in the app while other content was unnecessary or outdated. Thus, although it is difficult to develop user-friendly mobile apps 
due to the small screen size [41], it is possible to avoid such problems by presenting the content appropriately for the limited size. Considering this constraint, it is critical to design mobile apps as simply and plainly as possible when, at the same time, ensuring that they fulfill their basic functions. Every new function and feature to be added to the app should also be carefully considered not to complicate the app and adversely affect its usability [33].

By their nature, mobile apps need to provide short and fast interaction [41]. It is not possible to talk about the advantages of mobile apps if users cannot access necessary information quickly [43]. Studies have also shown that speed is an important factor in user preference to use mobile apps [e.g., 14]. Furthermore, freezing, constant errors, slowness and excessive battery consumption are among the main problems that lead users to give negative feedback to mobile apps [40]. Similarly, in the current study, some of the participants identified slowness as one of the main problems with the apps they evaluated. Apps being generally slow or some of the pages and processes being slow, particularly on certain days of the week were among the reported issues.

\subsection{Behavioral Strategies against Problems Encountered}

In a study investigating the responses of users in relation to an app not meeting their expectations and needs [40], the most utilized behavioral strategies were to uninstall the app immediately, remove it if it did not respond for longer than 30 seconds, tell their friends how bad it is, and complain about it in social media. In the current study, the participants developed two basic behavioral strategies to counter the problems with the mobile apps they evaluated; either trying to overcome the problem by continuing to use it, or giving up using the app and searching for alternatives.

Observing the relationship between the mobile app problems reported by the participants and the behavioral strategies they exhibited, it is clear that the types of problems differentiate the methods of handling the problems and the behavioral strategy adopted. The participants that chose the behavior to abandon the app often referred to functional errors, a feature that frustrated them, or the slow application speed. On the other hand, those participants that responded by continuing to use the app and trying to find some solution mostly mentioned problems with the interface design, ease-of-use, aesthetic characteristics, missing features or problems with the content of the app. Briefly, functional errors, disturbing features or slowness usually draw users away from the app. Although users that experienced design problems, missing features, or problematic content tended to give the evaluated apps another chance, the increasing competition in the mobile market and the availability of a large number of alternatives make it necessary to pay attention to such user complaints.

Users that are not satisfied with an app usually comment on it negatively to friends or colleagues [40]. Accordingly, an app with user-friendly features leads people to continue to use it and increase their possibility to recommend it to others [15, 25]. Some unsatisfied users not only remove an app but also make negative comments and reviews about the app, which can be influential in the download behavior of other users [44]. It 
is therefore important that mobile apps resolve both functional and design-related problems in order to have a larger share in the market, grow their target groups, and build a loyal customer base.

\section{Conclusion, Limitations and Future Research}

Today, mobile technologies continue to be used with increasing popularity. Accordingly, studies on the analysis of user behavior in the context of these technologies provide valuable findings. In this study, mobile app problems were examined from the point of view of university students, and the behavioral strategies they developed to counter these problems were also analyzed. The results revealed that the participants who encountered problems that made it difficult to use the app adopted the behavior to abandon the app whereas those that had complaints about design issues made more effort to continue to use the app. The data was collected from a limited number of participants. In terms of the generalizability of the findings, it is important that the data be obtained in a way to cover a broader group of participants. The participants were free to choose the app to evaluate, however we asked them to evaluate free and hedonicoriented apps. Therefore, an assessment based on the other app types (such as utilitarian-oriented apps) was not possible. Therefore, it is important that future work consider both the number of participants and the types of mobile apps. As the continuation of the current work, we plan to undertake further research in view of these issues.

\section{Acknowledgements}

We thank Dr. Frode Guribye, who provided helpful comments on previous version of this document.

\section{References}

1. Gomez, R.Y., Caballero, D.C., Sevillano, J.L.: Heuristic evaluation on mobile interfaces: A new checklist. The Scientific World Journal, Article ID 434326 (2014).

2. El-Kiki, T., Lawrence, E.: Emerging mobile government services: strategies for success. 20th Bled eConference eMergence: Merging and Emerging Technologies, Processes, and Institutions, Bled, Slovenia, pp. 776-788 (2007).

3. Erdem H., Türen, U., Kalkın G.: Mobil telefon yoksunluğu korkusu (nomofobi) yayılımı: Türkiye'den üniversite öğrencileri ve kamu çalışanları örneklemi. International Journal of Informatics Technologies, 10(1), 1-12 (2017).

4. Hsu, C.L., Lin, J.C.C.: What drives purchase intention for paid mobile apps? - An expectation confirmation model with perceived value. Electronic Commerce Research and Applications, 14, 46-57 (2015).

5. Statista: https://www.statista.com/statistics/276623/number-of-apps-available-in-leadingapp-stores/. (2017).

6. Dunn, B. K., Galletta, D. F., Hypolite, D., Puri, A., Raghuwanshi, S.: Development of smart phone usability benchmarking tasks. In System Sciences (HICSS), 2013 46th Hawaii International Conference on, pp. 1046-1052 (2013). 
7. Lee, G., Raghu, T.S.: Product portfolio and mobile apps success: evidence from app store market. Proceedings of the Seventeenth Americas Conference on Information Systems - All Submissions. 444 (2011).

8. Voas, J., Michael, J.B., van Genuchten, M.: The mobile software app takeover. IEEE Software, July/August, 25-27 (2012).

9. Zhang, D., Adipat, B.: Challenges, methodologies, and issues in the usability testing of mobile applications. International Journal of Human-Computer Interaction, 18(3), 293-308 (2005).

10. Hussain, A., Hashim, N.L., Nordin, N., Tahir, H.M.: A metric-based evaluation model for applications on mobile phones. Journal of ICT, 12, 55-71 (2013).

11. Miah, S.J., Gammack, J., Hasan, N.: Extending the framework for mobile health information systems research: A content analysis. Information Systems (2017).

12. Iacob, C., Harrison, R.: Retrieving and analyzing mobile apps feature requests from online reviews. 10th IEEE Working Conference on Mining Software Repositories, pp. 41-44 (2013).

13. Fu, B., Lin, J., Li, L., Faloutsos, C., Hong, J., Sadeh, N.: Why people hate your app - making sense of user feedback in a mobile app store. KDD'13, 1276-1284 (2013).

14. Bowen, K., Pistilli, M.D.: Student preferences for mobile app usage. EDUCAUSE Center for Applied Research (2012).

15. Chang, T.R., Kaasinen, E., Kaipainen, K.: What influences users' decisions to take apps into use? a framework for evaluating persuasive and engaging design in mobile apps for wellbeing. MUM'12 (2012).

16. Taylor, D.G., Levin, M.: Predicting mobile app usage for purchasing and information-sharing. International Journal of Retail \& Distribution Management, 42(8), 759-774 (2014).

17. Miniukovich, A., De Angeli, A.: Visual impressions of mobile app interfaces. NordiCHI'14, pp. 31-40 (2014).

18. Baharuddin, R., Singh, D., Razali, R.: Usability dimensions for mobile applications-A review. Research Journal of Applied Sciences, Engineering and Technology, 5(6), 2225-2231 (2013).

19. Kim, E., Lin, J.S., Sung, Y.: To app or not to app: engaging consumers via branded mobile apps. Journal of Interactive Advertising, 13(1), 53-65 (2013).

20. Harris, M.A., Brookshire, R., Chin, A.G.: Identifying factors influencing consumers' intent to install mobile applications. International Journal of Information Management, 36, 441450 (2016).

21. Chou, C.H., Chiu, C.H., Ho, C.Y., Lee, J.C.: Understanding mobile apps continuance usage behavior and habit: an expectance-confirmation theory. PACIS 2013 Proceedings. 132 (2013).

22. Wang, H.Y., Liao, C., Yang, L.H.: What affects mobile application use? The roles of consumption values. International Journal of Marketing Studies, 5(2), 11-22 (2013).

23. Maghnati, F., Ling, K.C.: Exploring the relationship between experiential value and usage attitude towards mobile apps among the smartphone users. International Journal of Business and Management, 8(4) (2013).

24. Le, T.D., Nguyen, B.T.H.: Attitudes toward mobile advertising: A study of mobile web display and Mobile app display advertising. Asian Academy of Management Journal, 19(2), 87-103 (2014).

25. Kim, S., Baek, T.H., Kim, Y.K., Yoo, K.: Factors affecting stickiness and word of mouth in mobile applications. Journal of Research in Interactive Marketing, 10(3), 177-192 (2016).

26. Huy, N.P., vanThanh, D.: Evaluation of mobile app paradigms. MoMM2012, pp. 25-30 (2012). 
27. Khalid, H., Shihab, E., Nagappan, M., Hassan, A.E.: What do mobile app users complain about? IEEE Software, May/June, 70-77 (2015).

28. Flood, D., Harrison, R., Iacob, C., Duce, D.: Evaluating mobile applications: A spreadsheet case study. International Journal of Mobile Human Computer Interaction, 4(4), 37-65 (2012).

29. Quettra Study: https://xupler.com/2016/11/03/mobile-app-uninstall-ratewhats-consideredgood-and-bad/ (2016)

30. Hazarika B., Khuntia J., Parthasarathy M., Karimi J.: Do Hedonic and Utilitarian Apps Differ in Consumer Appeal?. In: Sugumaran V., Yoon V., Shaw M. (eds) E-Life: Web-Enabled Convergence of Commerce, Work, and Social Life. WEB 2015. Lecture Notes in Business Information Processing, vol 258. Springer, Cham (2016).

31. Biel, B., Grill, T., Gruhn, V.: Exploring the benefits of the combination of a software architecture analysis and a usability evaluation of a mobile application. The Journal of Systems and Software, 83, 2031-2044 (2010).

32. Heidmann, F., Hermann, F., Peissner, M.: Interactive maps on mobile, location-based systems: design solutions and usability testing. Proceedings of the 21 st International Cartographic Conference (ICC), pp.1299-1306 (2003).

33. Harrison, R., Flood, D., Duce, D.: Usability of mobile applications: literature review and rationale for a new usability model. Journal of Interaction Science, 1(1) (2013).

34. Arsand, E., Froisland, D.H., Skrovseth, S.O., Chomutare, T., Tatara, N., Hartvigsen, G., Tufano, J.T.: Mobile health applications to assist patients with diabetes: lessons learned and design implications. Journal of Diabetes Science and Technology, 6(5), 1197-1206 (2012).

35. Mattson, D.C.: Usability assessment of a mobile app for art therapy. The Arts in Psychotherapy, 43, 1-6 (2015).

36. Nimbalkar, R.R.: Mobile application testing and challenges. International Journal of Science and Research, 2(7), 56-58 (2013).

37. Gao, J., Bai, X., Tsai, W., Uehara, T.: Mobile application testing: A tutorial. Computer, 4655 (2014).

38. Joorabchi, M.E., Mesbah, A., Kruchten, P.: Real challenges in mobile app development. ACM / IEEE International Symposium on Empirical Software Engineering and Measurement, 15-24 (2013).

39. Leontiadis, I., Efstratiou, C., Picone, M., Mascolo, C.: Don't kill my ads! Balancing privacy in an ad-supported mobile application market. HotMobile'12, San Diego, CA, USA (2012).

40. Apigee Survey: Users reveal top frustrations that lead to bad mobile app reviews. https://apigee.com/about/press-release/apigee-survey-users-reveal-top-frustrations-leadbad-mobile-app-reviews/ (2012).

41. Holzinger, A., Errath, M.: Mobile computer Web-application design in medicine: some research based guidelines. Universal Access in the Information Society, 6(1), 31-41 (2007).

42. Fung, R.H.Y., Chiu, D.K.W, Ko, E.H.T, Ho, K.K.W, Lo, P.: Heuristic usability evaluation of University of Hong Kong Libraries' mobile website. The Journal of Academic Librarianship, 42, 581-594 (2016).

43. Chae, M., Kim, J.: Do size and structure matter to mobile users? An empirical study of the effects of screen size, information structure, and task complexity on user activities with standard web phones. Behaviour \& Information Technology, 23(3), 165-181 (2004).

44. Inukollu, V.N., Keshamoni, D.D, Kang, T., Inukollu, M.: Factors influencing quality of mobile apps: Role of mobile app development life cycle. International Journal of Software Engineering \& Applications, 5(5), 15-34 (2014). 